\title{
Simulating hadron test beams in liquid argon
}

\author{
Alexander Friedland $\odot^{*}$ and Shirley Weishi Li $\odot^{\dagger}$ \\ SLAC National Accelerator Laboratory, 2575 Sand Hill Road, Menlo Park, California 94025, USA
}

(Received 3 August 2020; accepted 14 October 2020; published 9 November 2020)

\begin{abstract}
Thorough modeling of the physics involved in liquid argon calorimetry is essential for accurately predicting the performance of DUNE and optimizing its design and analysis pipeline. At the fundamental level, it is essential to quantify the detector response to individual hadrons-protons, charged pions, and neutrons-at different injection energies. We report such a simulation, analyzed under different assumptions about event reconstruction, such as particle identification and neutron detection. The role of event containment is also quantified. The results of this simulation can help inform the ProtoDUNE testbeam data analysis, while also providing a framework for assessing the impact of various cross section uncertainties.
\end{abstract}

DOI: 10.1103/PhysRevD.102.096005

\section{INTRODUCTION}

Energy resolution and the accuracy of energy scale calibration are essential characteristics for a neutrino detector operating in a broad-spectrum neutrino beam. Modeling these characteristics for the Deep Underground Neutrino Experiment (DUNE) is a nontrivial task. At the root of the problem is the nature of the final states produced when neutrinos of several-GeV energies interact with argon nuclei. These interactions can produce multiple hadrons of different types, which can, in turn, undergo subsequent interactions in the detector medium, distributing energy among even more particles. At first sight, by collecting all ionization charges, one should be able to measure all this energy calorimetrically. In reality, however, different particles create different amounts of detectable charge per unit energy lost, and some energy goes into invisible channels, such as nuclear breakup. As an extreme case, some or all neutrons may be altogether missed. Thus, having an accurate model for the detector response to each particle type is essential for optimal detector performance.

Given the complexity of the problem, a consistent way to study it is to simulate a large number of fully developed neutrino events [1,2]. The simulation pipeline in this approach combines a code modeling the primary neutrino interaction with another one propagating all resulting particles through the liquid argon medium. For the first

\footnotetext{
*alexfr@slac.stanford.edu

†shirleyl@slac.stanford.edu
}

Published by the American Physical Society under the terms of the Creative Commons Attribution 4.0 International license. Further distribution of this work must maintain attribution to the author(s) and the published article's title, journal citation, and DOI. Funded by SCOAP . code, one can use GENIE, GiBUU, or another event generator. For the second, the choices are GEANT4 or FLUKA, both of which model not only ionization losses but also any subsequent hadronic and electromagnetic interactions of all particles in the detector. The process needs to be repeated for different flavors of the incoming neutrino and a range of energy of interest. The result is a set of migration matrices describing probabilities connecting true and reconstructed energies. These matrices are an essential input for any analysis of oscillation sensitivity.

All this computer-intensive process is necessary just to characterize energy resolution in the case of baseline assumptions about the detector performance. If one wishes to investigate the impact of various changes to the reconstruction procedure, one needs to rerun the entire simulation pipeline. For example, one may wish to vary detection thresholds, exclude certain particle types, or investigate the impact of various particle identification (PID) assumptions. In each case, one obtains a new set of migration matrices, which then can be used for oscillation studies. An example study following this approach is presented in Ref. [2], where we considered several model assumptions about the detector performance, specifically, on the values of particle detection thresholds and the availability of accurate PID information.

To gain more insight into the physics dictating neutrino detection in liquid argon, in this paper, we inject in our simulation volume individual hadron particles-protons, charged pions, and neutrons-and investigate the detector response in each case. This should allow one to understand the role of each particle type in neutrino energy reconstruction. Of particular interest is quantifying the importance of reconstructing secondary neutrons.

There are several additional reasons to consider this study. First, our simulations yield "virtual test-beam data," which can be used to compare with the actual ProtoDUNE 
[3] test-beam data, an essential step to validating the entire simulation framework for the full events.

Second, it may also be used to devise sanity checks for the full event simulation results. Such checks are always necessary when one deals with large simulation frameworks with complex codes.

Third, one can use the results on individual particles to create simplified, flexible codes, in which prescriptions describing the detection process are applied to the outputs of the neutrino event generator. This is the general philosophy of the FASTMC code employed in the DUNE Conceptual Design Report (CDR) documents [4,5]. We regard this approach as very useful for certain problem types and far from being completely superseded by the full simulations. In connection with this point, it is extremely important to establish under which conditions the reconstructed energy for a given hadron type may be described by a Gaussian.

In our study here, we do not directly focus on detector signatures of electrons, muons, or gamma rays. The reason is based on our findings in Ref. [2]. Despite the different event topologies - muons leave long tracks, while electrons and gamma rays create electromagnetic showers-in all three cases, the total ionization charge was found to be in close correspondence with the true particle energy. Thus, the experimental energy resolution for these particles will likely be controlled by the reconstruction algorithm performance and not by physical processes in particle propagation, which are the focus of the present study.

The presentation is organized as follows. Section II presents an overview of our simulation framework and a list of specific reconstruction assumptions considered in the paper. Section III presents the simulation results for each hadron type. Section III A treats protons and also explains the reconstruction procedure. Section III B treats charged pions, while Sec. III C is devoted to the study of neutrons. Section IV explores the impact of limited detector volume. Section V discusses some consequences of the results of our study, including the physics dictating the energy resolution and possible applications to the development of simplified codes. Finally, Sec. VI summarizes our main findings.

\section{SIMULATION OVERVIEW}

Following Ref. [2], our simulations here also employ FLUKA [6,7] to model event development in liquid argon. FLUKA-here, we use version 2011.2x.6-is a publicly available, well-tested package that incorporates all relevant physics processes, such as ionization and radiative energy losses, hadronic inelastic interaction, and particle decays. Among its many strengths is a good description of $\mathrm{MeV}$ hadronic physics, as recently demonstrated by the ArgoNeuT experiment [8].

As in Ref. [2], we fully propagate all particles, including those produced in secondary interactions, but do not consider detector-specific effects, such as the finite lifetime of drifted charges, space charge distribution, wire spacing, electronic noise, or cosmogenic and radiogenic backgrounds. Such studies are beyond the scope of the present paper and will depend on specific detector configurations and performance characteristics. We are encouraged, however, by the extremely low levels of electronic noise in the ProtoDUNE-SP data [3] and assume that the reported issues with the space charge distribution will be adequately resolved.

Our emphasis at present is on assessing the physical impact of different reconstruction assumptions. Specifically, we aim to elucidate the impact of good PID and neutron detection. We argued in Ref. [2] that these are crucial factors determining the accuracy of neutrino energy measurements in liquid argon. Here, we deconstruct the argument by considering the reconstruction process for each hadron type. Accordingly, we analyze three model scenarios:

(1) Best reconstruction.-One has PID information on all charged particles in an event and applies it to get the ionization energy loss along each trajectory. The detection thresholds are considered to be very low, motivated by the ArgoNeuT experiment.

(2) Charge-only reconstruction.-No PID information is available for any secondary particles in an event. One collects the total ionization charge and uses it to infer, statistically, the energy of the injected particle.

(3) Charge-only, no neutrons. - In addition to the assumption of no PID, any energy imparted to neutrons at any stage in the process development is considered to be completely lost.

The first two scenarios were already considered in Ref. [2]. The second method is described in detail in Refs. $[9,10]$ and is currently accepted within the DUNE Collaboration as a way of treating the hadronic system [11]. The two scenarios represent extreme approaches to the treatment of PID information and thus allow us to quantify the impact of good PID on energy resolution. Realistic reconstruction efforts should produce an answer that is intermediate between the two scenarios, by reconstructing some of the particles, and the goal is to inform such efforts on how much can be gained by improved reconstruction.

The significance of these assumptions becomes obvious when one considers the anatomy of a hadronic event in liquid argon. Slower particles create more dense charge tracks, which, in turn, leads to more charge loss to recombination. Thus, a relationship between the detected charge, $d Q / d x$, and the true ionization energy loss, $d E / d x$, depends on the particle type. This is illustrated in Fig. 1, which shows what fraction of the ionization charge, created by protons, muons, and charged pions, of varying energies, is lost to recombination. The main physical effect here is that protons, being more massive, deposit more dense charge tracks, where more charge recombination takes place. As a corollary, protons yield less observable charge per unit energy lost than charged pions or muons of the same kinetic energy. 


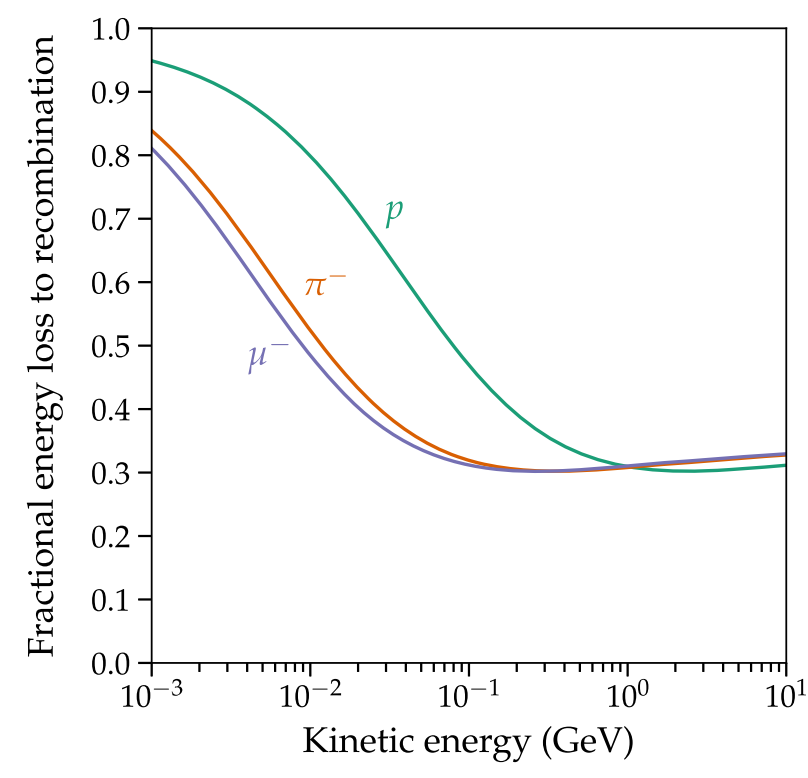

FIG. 1. Fraction of the ionization charge lost to recombination as a function of the kinetic energy of the particle, for protons, charged pions, and muons.

The third scenario is also introduced here as a deliberately extreme case. It allows us to quantify the importance of detecting neutrons. Neutrons, being electrically neutral, do not leave tracks at all. Their presence can be detected by the secondary ionizing particles they produce in their interactions. Since these interactions occur some distance away from their starting points, one ends up with secondary proton and pion tracks separated from the main event, and with a spray of small charge deposits created by the deexcitation gamma rays, from multiple disrupted nuclei, undergoing repeated Compton scattering $[2,12]$. The detectability of these different signatures will depend on a number of experimental factors-including reconstruction performance, containment issues due to finite detector volume, electronic noise, and radiogenic and cosmogenic backgrounds - and may be different for the near and far detectors. Nevertheless, it is highly reassuring that ArgoNeuT has demonstrated good ability to detect charge "blips" from nuclear deexcitations [8], while MicroBooNE has done the same for ${ }^{39} \mathrm{Ar}$ beta decay products [13]. So, once again, a realistic treatment of neutrons is expected to be somewhere between scenarios 1 and 3 .

In summary, the three scenarios outlined above are chosen to quantify the impact of the two main physical aspects of event reconstruction: the efficiencies fo PID and of neutron detection.

\section{SIMULATION RESULTS}

\section{A. Protons}

We begin by considering proton beams. We will use this case to describe our simulation procedure and to explain how we use its results to model energy resolution.
First, we generate our simulation dataset, which is used in each of our three scenarios. For this, we inject protons of energies from 0.01 to $3.0 \mathrm{GeV}$ into unlimited liquid argon volume and model the full event development in each case. The effects of finite detector size deserve a dedicated discussion, and we will address them in Sec. IV below. Between 0.1 and $3.0 \mathrm{GeV}$, we sample proton energy values in $0.05 \mathrm{GeV}$ intervals. To better characterize the resolution at low energies, we also generate the second dataset with $0.01 \mathrm{GeV}$ energy spacing. For each value of the true energy, $E_{\text {tr }}$, we generate $10^{4}$ events.

For definiteness, let us for now specialize to the totalcharge study (scenario 2). The simulation dataset tells us, for a given value of the true proton energy, $E_{\text {tr }}$, the probability $P\left(Q \mid E_{\text {tr }}\right)$ of measuring charge $Q$. Discretizing (binning) the $Q$ values, we obtain a matrix of probabilities connecting $E_{\text {tr }}$ and $Q$. Explicitly, the matrix element $P\left(Q^{(j)} \mid E_{\text {tr }}^{(i)}\right)$ equals the number of events that landed in bin $Q^{(j)}$ divided by the number of simulations with $E_{\mathrm{tr}}^{(i)}$. This matrix, in the graphical form, is presented in Fig. 2. The left panel covers the full range of $E_{\text {tr }}$, up to $3 \mathrm{GeV}$; the right panel depicts the finely sampled low-energy dataset, covering $E_{\text {tr }}$ values up to $0.5 \mathrm{GeV}$. The width of the bins in $Q$ is the same as the corresponding $E_{\text {tr }}$ bins: $0.05 \mathrm{GeV}$ in the first simulation and $0.01 \mathrm{GeV}$ in the second one.

Now, suppose we use this simulation dataset to analyze a new event, created by a proton with an unknown value of $E_{\text {tr. }}$. Given the value of $Q$ measured for this event, we can use our matrix as a lookup table, to obtain the probability $P\left(E_{\text {rec }} \mid Q\right)$ that the event was created by a proton with energy $E_{\mathrm{rec}}$. Explicitly, $P\left(E_{\mathrm{rec}}^{(j)} \mid Q^{(i)}\right)$ is equal to the number of times charge $Q^{(i)}$ was obtained in the simulation with proton energy $E_{\text {rec }}^{(j)}$ divided by the total number of times charge $Q^{(i)}$ was obtained for all energies in our simulation set.

What we just described provides a reconstruction procedure, which allows one to go from the detected value of $Q$ to $E_{\text {rec }}$. The requirement of unbiased reconstruction is assured by construction, since our proton energy values are drawn from a flat distribution. This procedure is designed to capture the reconstruction problem for an actual (nontest-beam) experiment, where, in a most general case, one does not have any prior information on the distribution from which proton energies are drawn. For example, one may have a proton track in the middle of a complicated neutrino event.

In summary, while reading the matrix in Fig. 2 "vertically" gives us a probability distribution of ionization charge $Q$ for a proton of a known $E_{\text {tr }}$, reading the same matrix "horizontally," with appropriate normalization, provides an unbiased reconstruction procedure.

We now have all ingredients to describe our full energy resolution modeling pipeline. To this end, it is important to precisely define what we mean by "energy resolution." 

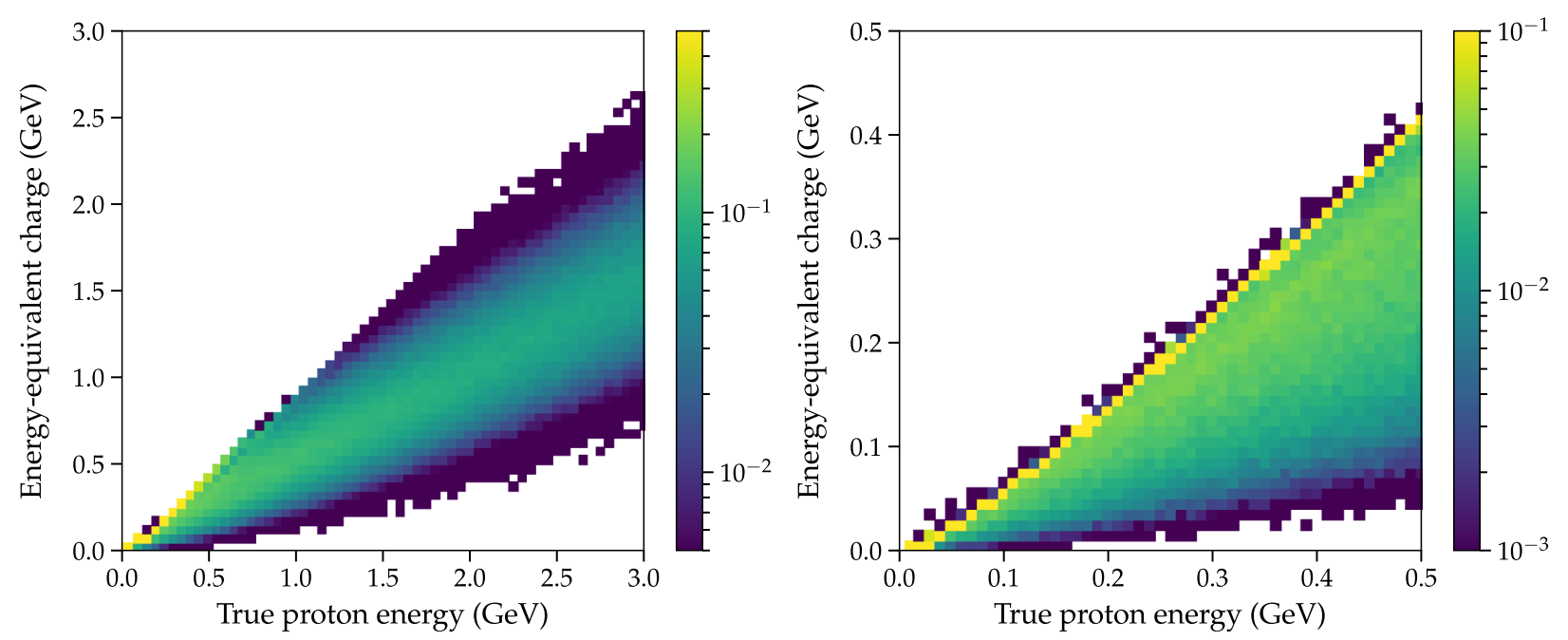

FIG. 2. Graphical representation of the matrix connecting ionization charge $Q$ and the true energy of the injected proton $E_{\mathrm{tr}}$, according to our simulations. (Left) $E_{\mathrm{tr}}$ from 0 to $3 \mathrm{GeV}$ and (right) $E_{\mathrm{tr}}$ from 0 to $0.5 \mathrm{GeV}$ at finer numerical sampling. The color of each square indicates the probability of obtaining the corresponding interval of charge $Q$ given the value of $E_{\text {tr }}$.

Loosely speaking, this term could simply refer to the width of the $Q$ distribution that is obtained when a beam of a given energy $E_{\mathrm{tr}}$ is shot into the detection volume. More accurately, energy resolution in this paper will describe the width of the distribution $P\left(E_{\text {rec }} \mid E_{\text {tr }}\right)$, of reconstructed energies $E_{\text {rec }}$ obtained starting with $E_{\text {tr }}$ and reconstructing each event independently, using the lookup procedure described above.

To find the probability distribution of reconstructed energies, $P\left(E_{\text {rec }} \mid E_{\text {tr }}\right)$, we must sum (integrate) over all $Q$ values that can be obtained in the intermediate step:

$$
P\left(E_{\mathrm{rec}} \mid E_{\mathrm{tr}}\right)=\int d Q P\left(E_{\mathrm{rec}} \mid Q\right) P\left(Q \mid E_{\mathrm{tr}}\right)
$$

It can be straightforwardly shown that, if the charge distribution is Gaussian,

$$
P\left(Q \mid E_{\text {tr }}\right)=\frac{1}{\sqrt{2 \pi} \sigma} \exp \left[-\frac{\left(Q-E_{\mathrm{tr}} f\right)^{2}}{2 \sigma^{2}}\right],
$$

where $f$ is the fraction of energy that on average goes into charge, the resulting distribution of $E_{\text {rec }}$ is also Gaussian, with the width $\sqrt{2} \sigma / f$ :

$$
\begin{aligned}
P\left(E_{\mathrm{rec}} \mid E_{\mathrm{tr}}\right) & =\int d Q P\left(E_{\mathrm{rec}} \mid Q\right) P\left(Q \mid E_{\mathrm{tr}}\right) \\
& =\frac{f}{2 \sqrt{\pi} \sigma} \exp \left[-\frac{\left(E_{\mathrm{rec}}-E_{\mathrm{tr}}\right)^{2} f^{2}}{4 \sigma^{2}}\right] .
\end{aligned}
$$

Here, the probability distribution $P\left(E_{\text {rec }} \mid Q\right)$ is given by

$$
P\left(E_{\mathrm{rec}} \mid Q\right)=\frac{f}{\sqrt{2 \pi} \sigma} \exp \left[-\frac{\left(Q-E_{\mathrm{rec}} f\right)^{2}}{2 \sigma^{2}}\right],
$$

which is normalized to 1 . In a general case, however, the distributions for $E_{\text {rec }}$ and $Q$ do not follow the same functional form.

The application of this procedure to the other two reconstruction methods is now straightforward. For the simulation with no neutrons, all charges created downstream of any neutron are discarded, with the rest of the procedure unaffected. In the best-reconstruction case, to each track in the event, we apply a charge recombination correction factor that is a function of its PID. The resulting distribution of the "modified charged" is used in place of $Q$.

Figure 3 shows the result of applying this procedure to our simulation set. Four representative values of the true proton energy are considered: $0.1,0.5,1.0$, and $2.0 \mathrm{GeV}$. We see that the character of the distribution changes as one goes from low to high energy values: at $2 \mathrm{GeV}$, the $E_{\text {rec }}$ distributions are well described by Gaussians, while at $0.1 \mathrm{GeV}$, the distribution is dominated by a sharp spike, where essentially all proton energy is recovered. The $0.5 \mathrm{GeV}$ represents a transition between these regimes. This transition is also clearly seen in Fig. 2, where the yellow diagonal points represent unscattered protons.

The change from the unscattered to multiply scattered regimes is dictated by the mean free path of hadronic collisions. It will prove crucial for our discussion in Sec. V below. But first, we turn to the corresponding results for the other hadrons.

\section{B. Charged pions}

Understanding the propagation of charged pions is also of direct relevance to DUNE calorimetry. As illustrated in Ref. [2], interactions of $4 \mathrm{GeV}$ neutrinos can create hadronic showers with multiple pions, with energies in the hundreds of $\mathrm{MeV}$ range. Even 1-2 $\mathrm{GeV}$ pions are not uncommon in 

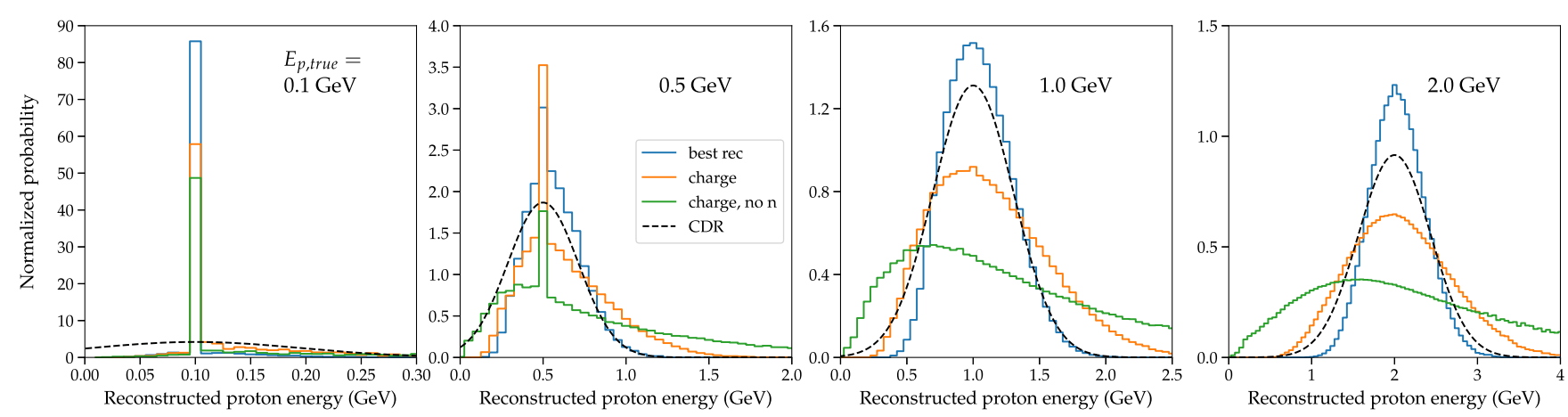

FIG. 3. Distributions of proton reconstructed energies, for four representative values of the true energy, $E_{p}=0.1,0.5,1.0$, and $2.0 \mathrm{GeV}$. Three different reconstruction scenarios are considered: (i) full PID information is available ("best rec"), (ii) only total ionization charge ("charge"), and (iii) total ionization charge with neutrons undetected ("charge, no n"). For comparison, the dashed curve shows the resolution assumed in the DUNE CDR document $[4,5]$.

such events. Therefore, it is certainly worth considering charged pion test beams, and indeed ProtoDUNE has collected such data.

In Fig. 4, we simulate charged pion beams, with energies $0.2,0.5,1$, and $2 \mathrm{GeV}$. The histograms in the figure correspond to $\pi^{-}$; for positively charged pions, the results are very similar. The reconstruction assumptions and the analysis are the same as considered earlier for protons. We see that the basic results for pions and protons are qualitatively similar: the distributions of reconstructed energies are non-Gaussian at the lowest energies and become Gaussian at higher energies. One notable quantitative difference is that the Gaussianity sets in at a smaller energy for pions than for protons. This has a natural physical explanation in terms of the ionization rates in the two cases. With energy loss having a $v^{-2}$ leading velocity dependence, slower particles lose energy faster per unit distance traveled. Since protons of a few hundred $\mathrm{MeV}$ are nonrelativistic, their ionization rates are higher than for pions of the same kinetic energy. Thus, protons are more likely than pions to come to rest before undergoing hadronic interactions, and it is repeated hadronic interactions that create Gaussian distribution of reconstructed energies.

\section{Neutrons}

We have seen that neutron detection has a dramatic impact on the accuracy of the calorimetric energy reconstruction by liquid argon detectors. Let us now take a deeper dive into the subject by analyzing neutron propagation and interactions.

First of all, one should be more precise about what is meant by neutron detection. As already mentioned in Sec. II, a neutron traveling through the liquid argon medium does not, by itself, create an ionization track. Its energy is lost via interaction with multiple argon nuclei, and it is through the secondary particles created in these interactions that the presence of the neutron can be revealed. Importantly, the secondary charged particles carry only a fraction of the original neutron energy - some of the energy is lost to nuclear breakup. Hence, a direct calorimetric measurement of the neutron energy is not possible. One recovers only part of the energy and uses a simulationbased model to infer the likely energy range of the original neutron.

At a more detailed level, one has to consider the different signatures that can be created in neutron interactions. A neutron can excite an argon nucleus, or it can knock out one or more nucleons from it, leaving the daughter
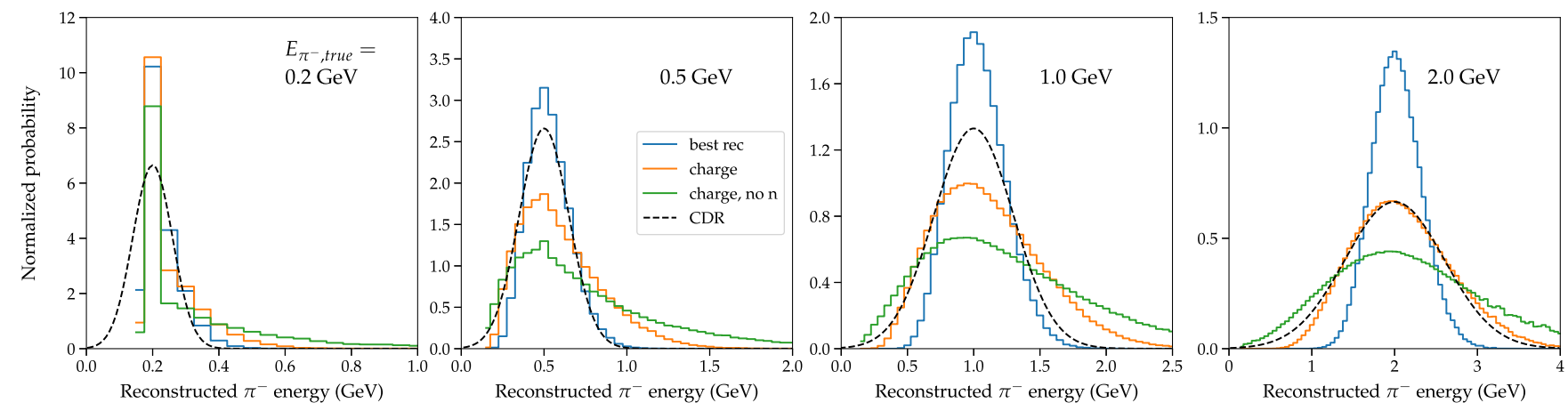

FIG. 4. Same as Fig. 3, but for the $\pi^{-}$reconstructed energies. 
nucleus in an excited state. The deexcitation gammas undergo Compton scattering in the medium, and the recoil electrons leave small ionization charge deposits [12]. Since a given neutron interacts with many argon nuclei in this way, many recoil electrons are scattered over an extended region. The resulting spray of such small charges, from many nuclear interactions, is, in principle, observable, as demonstrated by the ArgoNeuT analysis [8].

A more prominent signature comes from energetic knockout products. In particular, a sufficiently energetic proton can create a distinct track that is detached from the main event. Such tracks can be identified as protons, thus enabling proper charge recombination correction. In Fig. 5, we depict a spectrum of the leading (highest-energy) protons created in propagation of neutrons of two starting kinetic energies: 0.5 and $1 \mathrm{GeV}$. Estimating the threshold for proton identification to be $30 \mathrm{MeV}$, we see that a large fraction of the knockout protons could be identified.

This remains true even at lower neutron energy. For example, for a $300 \mathrm{MeV}$ neutron, on average, $34 \%$ of the energy goes into knockout protons above the $30 \mathrm{MeV}$ threshold, according to our simulations. An additional $4 \%$ of the energy goes into protons below that threshold; $40 \%$ is lost to nuclear breakup; $14 \%$ goes into gammas; $4 \%$ is imparted to heavy ions knocked out of the nuclei; $2 \%$ goes to nuclear recoil; and, finally, $2 \%$ goes to pions produced in hadronic collisions. Thus, the full energy budget is quite complicated, and the accuracy of energy reconstruction depends on how much of that energy can be recovered.

Three comments about these numbers are in order. First, the process is highly stochastic, and event-to-event variations are found to be large. For example, the energy fraction

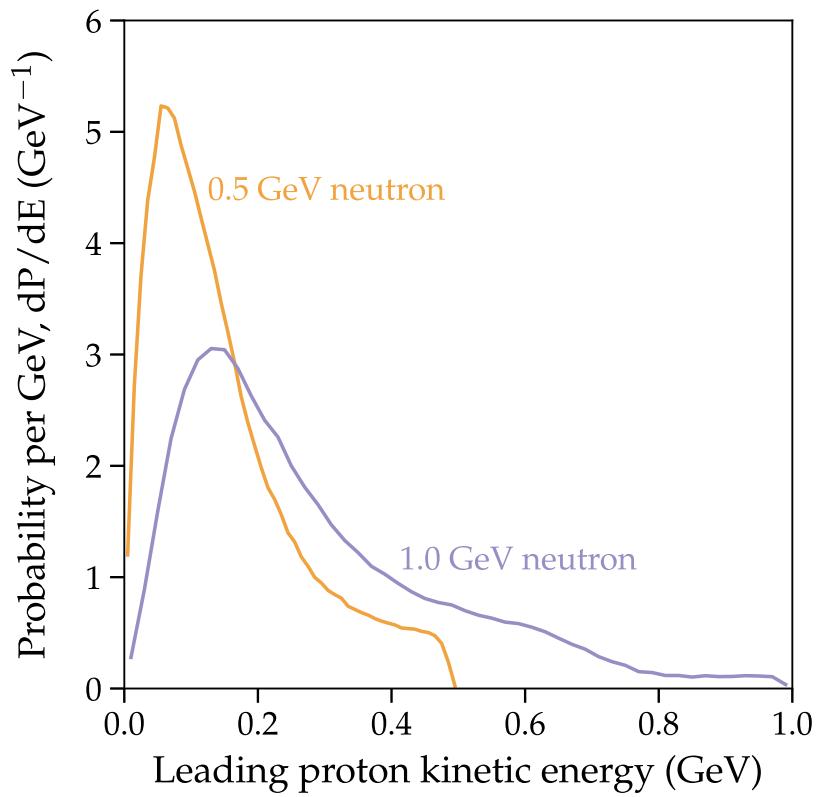

FIG. 5. Kinetic energy distributions of the most energetic protons produced by 0.5 and $1.0 \mathrm{GeV}$ neutrons. in the leading proton has a range of $38 \pm 24 \%$. Second, the fractions obviously change with neutron energy. In particular, inelastic hadronic interactions become more prominent at higher energy. For $1 \mathrm{GeV}$ neutrons, as much as $19 \%$ of the energy goes into pions. Third, the components in subthreshold protons and in heavy ions require special attention.

The subthreshold protons are those for which the reconstruction algorithm is not able to identify a clear track. In such a case, they appear as part of the spray. Compared to Compton-recoil electrons, however, which make up a lot of the charge blips in the spray, these low-energy hadrons are subject to larger charge recombination. Thus, if one wished to use the measured charge in the spray to improve the neutron energy reconstruction-compared to what is possible from the leading proton alone- the composition of the spray must be reliably understood. On the other hand, the charge blips due to low-energy protons should have a significantly higher charge concentration than the corresponding electron blips. We propose investigating if reconstruction algorithms could be taught to distinguish proton and electron blips based on the charge concentration, even when a definite track could not be identified.

Similar considerations also apply to the heavy ion products. Our simulation shows that most of such ions are deuterons, with the average kinetic energy of $64 \mathrm{MeV}$ at production. Taking the rates of charge recombination into account, a $64 \mathrm{MeV}$ deuteron deposits as much charge as a $53 \mathrm{MeV}$ proton, but with a $d E / d x$ energy loss rate that is approximately $50 \%$ greater. Hence, it might in principle be possible to train the reconstruction software to also identify most deuterons, further improving energy reconstruction.

For all these reasons, detailed analysis of the nature of the small charge deposits represents a potentially promising way to improve energy resolution of the liquid argon technology. The necessary studies on subthreshold protons and deuteron ions could be carried out at MiniBooNE and ProtoDUNE.

Finally, all results given here rely on the accuracy of the neutron interaction modeling in FLUKA, and direct neutron test-beam measurements are highly desirable to validate the simulations. In this case, we note two important, complementary experimental efforts. The first is with the miniCAPTAIN detector. This experiment already ran and collected data at the Los Alamos National Laboratory [14], in a neutron beam with energies between 100 and $800 \mathrm{MeV}$, but so far has only presented total cross section results. We encourage the collaboration to specifically analyze the distribution of the leading knockout proton energies.

The second is the calibration study that was conducted at ProtoDUNE this summer, using a $2.5 \mathrm{MeV}$ pulsed neutron source. These measurements should help validate the neutron transport and capture model, and we are eagerly awaiting the release of the results. 

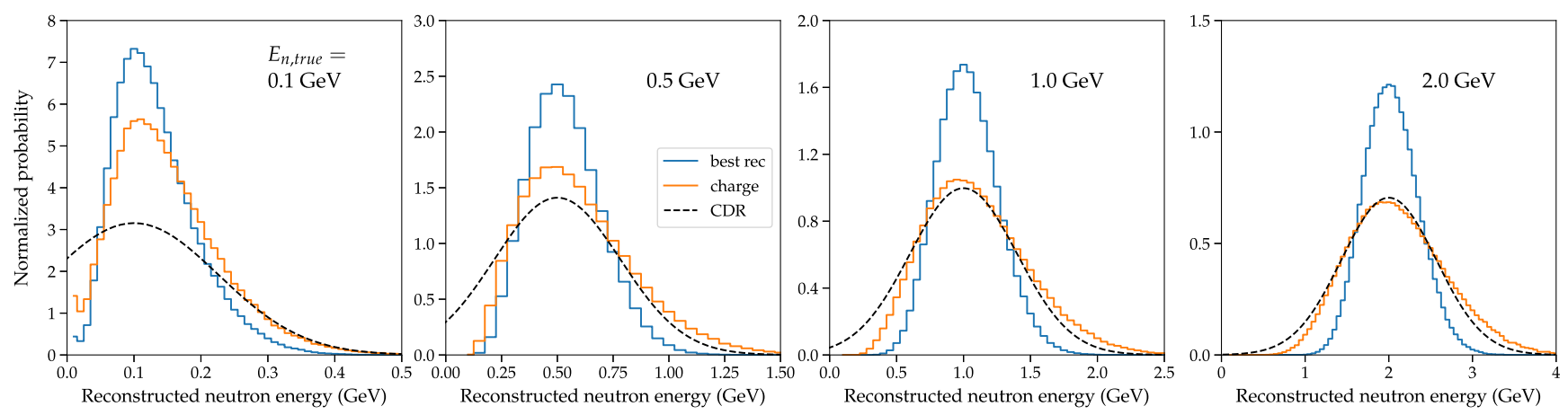

FIG. 6. Distributions of the neutron reconstructed energies, for four representative values of the true energy, $E_{n}=0.1,0.5,1.0$, and $2.0 \mathrm{GeV}$. Two different reconstruction scenarios are considered: (i) full PID information is available (blue) and (ii) only total ionization charge (orange). For comparison, the dashed curve shows the resolution assumed in the DUNE CDR document.

To this end, we simulate energy reconstruction expected from a neutron test beam. In Fig. 6, we present results for neutrons of initial energies of $0.1,0.5,1.0$, and $2.0 \mathrm{GeV}$. One of the most striking observations here is that at high energies, greater than or approximately equal to $1 \mathrm{GeV}$, the histograms of reconstructed energy begin to look quite similar to those of the charged hadrons, shown earlier. We will return to this important point in Sec. V.

\section{EFFECTS OF LIMITED VOLUME}

As the next step, we will consider what happens if the detection volume is limited. This study has two motivations. From the practical side, such a situation could be realized in ProtoDUNE [3], if one analyzes ionization charges collected in a single anode plane assembly, or light detected by a single light collection bar [15]. It may also have implications for the design of near detectors, as we noted in Ref. [2]. From the conceptual point of view, we would like to understand how the spatial development of the events impacts the accuracy of calorimetric measurements.

We consider proton beams with two initial energy values, 2 and $7 \mathrm{GeV}$. The first case is motivated by the relevance to the DUNE experiment, where the neutrino energy varies in the approximately $1-4 \mathrm{GeV}$ range. The second one occurs in ProtoDUNE, where the test-beam energies ran a range of values, including $7 \mathrm{GeV}$.

The simulation results are collected in Fig. 7, where the volumes considered are $2 \times 2 \times 2 \mathrm{~m}, 3 \times 3 \times 3 \mathrm{~m}$, and $5 \times 5 \times 5 \mathrm{~m}$, left to right. The top row corresponds to injected protons of $2 \mathrm{GeV}$ energy, and the bottom row shows the corresponding results for $7 \mathrm{GeV}$ protons. In each case, we consider the method of total charge calorimetry and bin the simulation results in "energy-equivalent charge," which is defined as energy lost by a minimally ionizing muon that creates the same amount of ionization charge. Specifically, one ionization electron is counted as $23.6 \mathrm{eV}$ of lost energy [2,3].

We see that, while in the $5 \times 5 \times 5 \mathrm{~m}$ volume the charge distribution closely follows a simple Gaussian shape, the situation in the smaller volumes is more complicated. In addition to the scattered component, we also clearly see an unscattered one. Given the mean free path for hadronic interactions approximately $80 \mathrm{~cm}$, the fraction of unscattered protons exiting the $2 \times 2 \times 2 \mathrm{~m}$ volume is approximately $\exp (-2 / 0.8) \sim 8 \%$, consistent with what is seen in the histogram.

The second relevant observation concerns the scattered component. The dashed curves in the top panels show the corresponding Gaussian fits. We see that, while the absolute width of the Gaussian stays approximately the same in all volumes, the center of the Gaussian moves to higher energies (charge) as the volume is increased. This indicates that in the smaller volumes the shower is not yet fully developed. For example, for $2 \mathrm{GeV}$ injected protons, about $22 \%$ of all ionization charges are created outside of the $2 \times 2 \times 2 \mathrm{~m}$ volume, and $8 \%$ are created outside of the $3 \times 3 \times 3$ m volume.

This is directly confirmed by examining the spatial distribution of the ionization charge in our simulation. Figure 8 shows the distribution of charges found after injecting $4 \times 10^{5}$ protons at position $(0,0,0)$. The initial proton kinetic energy is $2 \mathrm{GeV}$, and the momentum points along the $z$ direction. The $y$ coordinate has been suppressed, so the graphics shows the charge projection onto the $(x, z)$ plane. The contours show the regions enclosing 95\% and $99 \%$ of the total charge. The cubic volumes considered above are shown with dashed lines.

We clearly see that the smaller volumes fail to enclose the full charge distribution. Even the $5 \mathrm{~m}$ box misses a few percent of the ionization charge. These charges form an extended "halo" and are induced mostly by diffusing neutrons. Interestingly, some of the charge lies in the backward direction (at negative $z$ ). This charge cannot be captured at ProtoDUNE but may be detected in the DUNE far detector.

For $7 \mathrm{GeV}$ injected protons, the effects of the limited volume are even more pronounced, as indicated by an extended shoulder between the unscattered spike and the peak of the scattered distribution. 

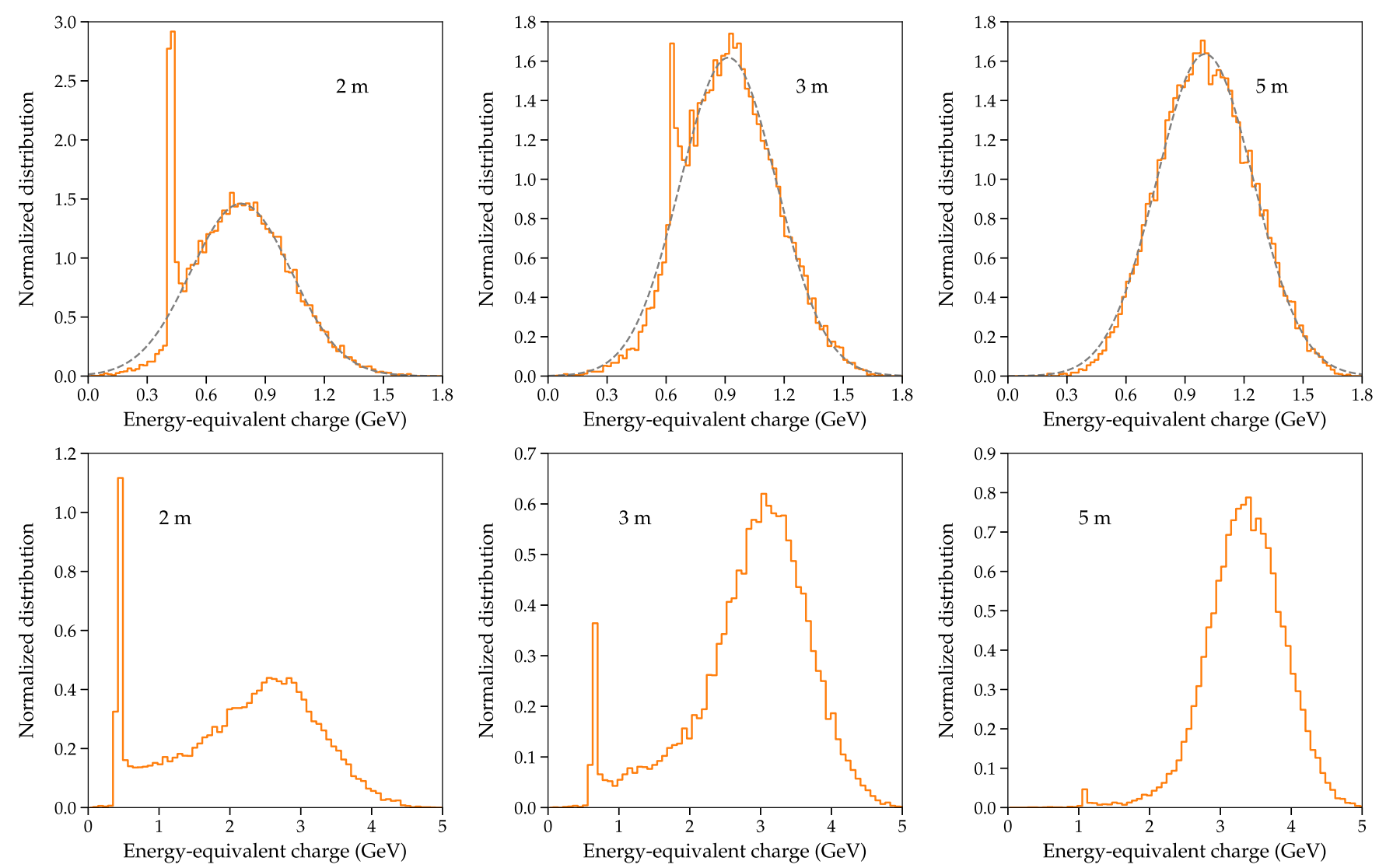

FIG. 7. Distribution of ionization charges created by an injected proton in cubic volumes of length 2,3 , and $5 \mathrm{~m}$. The top row corresponds to injected proton energy of $2 \mathrm{GeV}$; the bottom row, to proton energy of $7 \mathrm{GeV}$. The dashed curves in the top row show the corresponding Gaussian fits.

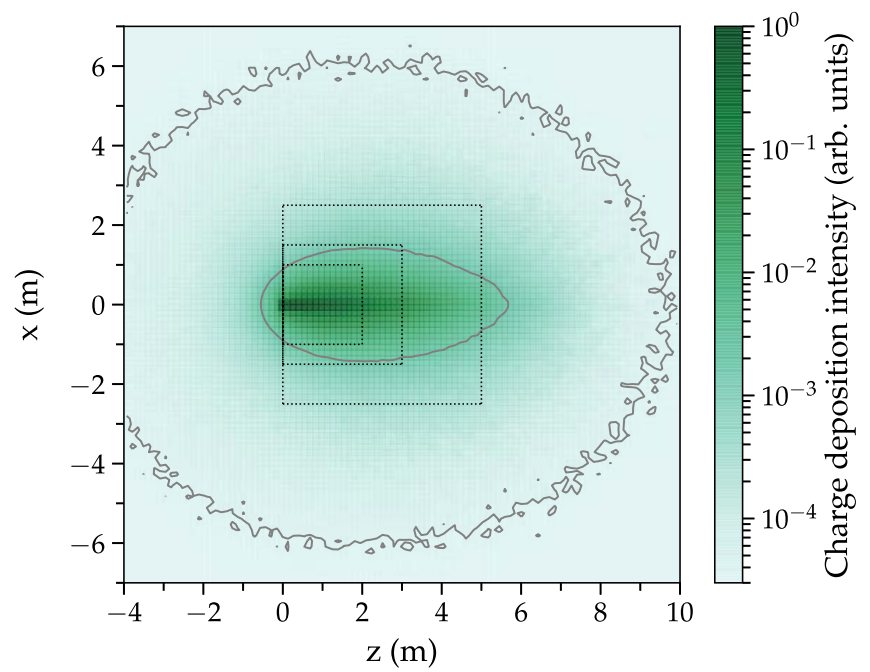

FIG. 8. Distribution of ionization charges created by injecting $4 \times 10^{5}$ protons of $2 \mathrm{GeV}$ kinetic energy at position $(0,0,0)$. The initial proton momenta point in the positive $z$ direction. All charges have been projected along the $y$ direction. The solid contours show the regions enclosing $95 \%$ and $99 \%$ of the total charge. The dashed lines show the $2 \times 2 \times 2 \mathrm{~m}, 3 \times 3 \times 3 \mathrm{~m}$, and $5 \times 5 \times 5 \mathrm{~m}$ cubic volumes considered in Fig. 7 .
This shows that behind seemingly simple Gaussian resolution curves seen in Sec. III lies a complicated dynamical picture of shower development. The resolution of a detector may thus be affected by its geometry and other relevant considerations, such as requirements to fiducialize the detection volume to eliminate cosmic ray-induced and other contamination.

\section{DISCUSSION}

The results of our large-volume simulations can be summarized by plotting the energy resolution for each particle type, as a function of energy. This is shown in Fig. 9, where injection energies up to $3 \mathrm{GeV}$ are considered. The colored curves correspond to the three reconstruction scenarios we consider, as labeled. The dashed curves indicate the resolution assumed in the CDR document $[4,5]$.

We immediately see that the role of neutrons is absolutely crucial for the accuracy of charge hadron energy reconstruction: the green curves, which correspond to discarding all neutrons, show the resolution that is significantly worse than the other two cases. This is in line with what we already discussed in Sec. III for specific energy values. Even though the average fraction of energy that 

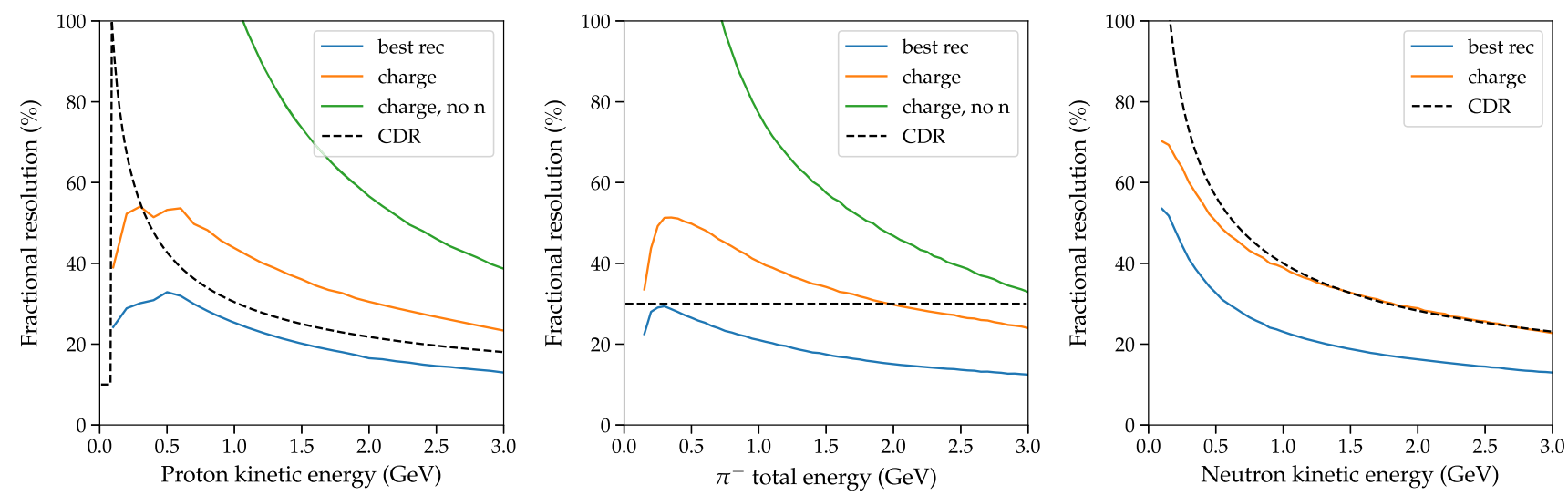

FIG. 9. Simulated hadron energy resolution as a function of its true energy. Left to right: protons, negative pions, and neutrons.

goes into secondary neutrons is quite stable, about $20 \%$, the event-by-event variation of this fraction is very large [2].

Let us now turn to the other two reconstruction scenarios. Notably, at sufficiently high energies, the fractional energy resolution is well fit by a $E^{-1 / 2}$ scaling law. Specifically, for protons, we obtain $42 \% / \sqrt{E}$ for the charge-only method and $25 \% / \sqrt{E}$ for the best-reconstruction method. For charged pions, we find $42 \% / \sqrt{E}$ for the charge-only method and $21 \% / \sqrt{E}$ for the best-reconstruction method. For neutrons, the corresponding relationships are 40\%/ $\sqrt{E}$ for the charge-only method and $23 \% / \sqrt{E}$ for the bestreconstruction method. The first observation, therefore, is that at high energies the energy resolution performance is remarkably similar for each particle type.

The second observation is that the $E^{-1 / 2}$ law breaks down at lower energies, and the fractional resolution actually improves as the energy is decreased to $0.1 \mathrm{GeV}$. Let us discuss the underlying reasons for this behavior.

At the most basic level, liquid argon detectors operate as calorimeters, in which ionization charge deposited by particles created as a result of neutrino interactions is used to infer the total energy. Conversion from charge to energy involves, however, a number of steps that each introduce uncertainty. The size of this uncertainty depends on the amount of additional information gained in the reconstruction process. Let us summarize the relevant factors:

(a) For a given final-state track, the first consideration is its PID. Conversion from charge deposited along a track to energy involves correctly accounting for charges lost to recombination. The recombination correction is higher for slow-moving protons than for pions and muons of the same kinetic energies, as illustrated in Fig. 1. One might wonder whether the discrimination between neutral and charged pions, i.e., between electromagnetic showers and tracks, also plays a role here. In fact, we explicitly checked that, in our simulation, the main impact of PID on the accuracy of energy reconstruction is through proton/ charged pion discrimination. (b) The next fundamental ingredient in the energy reconstruction of charged hadrons is their interactions in the medium. Indeed, once the particle type is identified, $d Q / d x$ along its trajectory can be reasonably well related to $d E / d x$, until the particle undergoes a hadronic interaction with a background argon nucleus.

In hadronic collisions, the energy flow is affected by several processes:

(i) Some energy is lost to the breakup of the target nucleus. Some can be emitted by deexcitation gamma rays, which create small charge deposits that may be detected with a varying degree of efficiency, depending on the detection thresholds.

(ii) Energy can be imparted to one or more hadrons, such as secondary pions created in the collision, nucleons knocked out of the nucleus, or a combination of pions and nucleons. For each secondary track, the accuracy of conversion from charge to energy loss again depends on whether PID information is available.

(iii) Some of the knocked-out nucleons in the last step could be neutrons, and these present a special challenge, as discussed in Sec. III C. They do not leave tracks and can dissipate energy by exciting and breaking up numerous argon nuclei, resulting in a spray of small charge deposits. They may also produce tertiary charged hadrons, which are likewise detached from the main event. Energy reconstruction depends on whether and how often such detached charge deposits can be identified with the main event.

Above all, the main conclusion here is this: the nature of the energy resolution is dictated by the frequency of hadronic collisions. Hadrons above $1 \mathrm{GeV}$ (and their products) are expected to undergo multiple collisions. In this regime, the distribution of energy among the several channels becomes stochastic, and the reconstructed energy distribution approaches a Gaussian form. Notice that the widths of the Gaussians, which have been derived earlier, are found to be very similar for the three hadron types. They are controlled by the similar hadronic interaction rates. 
On the other hand, at lower energies, the interactions are only sporadic, and the distributions of reconstructed energies become more and more asymmetric. The Gaussian width prescriptions obtained at higher energies break down at these energies. For protons of approximately $100 \mathrm{MeV}$ energy, the high-energy Gaussian width fails dramatically. Instead, the energy can be reconstructed with very good accuracy, assuming good PID.

As a corollary, for protons and charged pions, the worst relative resolution occurs at energies of several hundred $\mathrm{MeV}$, as seen in the graphs. We see that this behavior is not captured by the assumptions of the CDR (shown with dashed curves).

Given the crucial role of the hadronic interactions, it is essential that our predictions for them (made with FLUKA) be directed tested with ProtoDUNE. This applies not only to the frequency of collisions but also to the statistics of the final states produced.

Let us now consider some important applications for our results. Consider two types of problems:

(i) estimating the impact of various detector changessuch as gradually improving neutron detection efficiency, or improving PID;

(ii) understanding the impact of various cross section uncertainties, especially the impact of several continuously varied parameters in the model.

For example, suppose one considers changes to the pion production model for neutrino-nucleon interactions, to reduce the tensions with the electron scattering data [16]. This adjustment may result in the modification of the properties of the hadronic final states [17]. One would like to be able to gauge the impact of these changes on neutrino energy reconstruction, without having to regenerate the full event simulation set after each incremental adjustment, which carries prohibitive computing costs.

This calls for the need to build simplified codes, as we mentioned in the Introduction. Such codes would, instead of simulating full events in the detector, apply certain "smearing" prescriptions to the final-state particles output by the neutrino-nucleus event generator, in the spirit of FASTMC [4,5]. Such a framework would give approximate answers to the questions of energy resolution and energy scale calibration, in response to various assumptions about cross section physics or detector performance. It can also be used to explore sensitivity to various new physics scenarios.

Our virtual test-beam simulations provide crucial input into such a framework. As we saw, it gives not only the width of the distribution of reconstructed energy but also when the energy of a particle can be Gaussian smeared and when a different functional form must be used.

\section{CONCLUSIONS}

In summary, the two main lessons of our investigations in this paper are as follows. First, the energy resolution of liquid argon time-projection chamber detectors strongly depends on the detector parameters and performance. Among the relevant factors are the detector geometry, which may impact event containment, and the quality of event reconstruction. In particular, the inability to reconstruct detached charge deposits due to neutrons leads to a large resolution penalty.

Second, for hadrons with energies in the $\mathrm{GeV}$ range, the resulting distributions of reconstructed energies are often non-Gaussian. Namely, we have the following:

(i) With neutrons dropped, we consistently find a very non-Gaussian charge distribution, even when the detection volume is large.

(ii) Conversely, in a limited volume $(2 \times 2 \times 2 \mathrm{~m})$, for high initial energy, we again get a non-Gaussian charge distribution, even with neutrons included.

(iii) We have considered a total charge measurement with no PID corrections. In a large volume, with detached charges created by neutrons, the distribution starts approaching Gaussian at $2 \mathrm{GeV}$.

(iv) The best-case scenario is when the charges are collected over a large volume, neutron-induced charges are included, and full PID corrections are implemented. In this case, the distribution is Gaussian even at $1 \mathrm{GeV}$.

(v) Even in the best-case scenario, however, at low hadron energies, the distribution is always nonGaussian; this happens for proton energies less than or approximately equal to $0.6 \mathrm{GeV}$ and charged pion energies less than or approximately equal to $0.4 \mathrm{GeV}$.

We noted before [2] that loss of information about a neutrino-induced event always leads to worsening of energy resolution. We clearly see this here, at the level of individual hadrons. We also see that the same loss of information-either by failure to contain the full event or by missing some particles - often leads to non-Gaussianity of the reconstructed energy distribution.

We see that the situation is quite different from the case of highly energetic particles, where the corresponding hadronic shower can fully develop. In that case, numerous statistical fluctuations combine to make the calorimetrically determined energy fluctuate in an approximately Gaussian manner. In the range of energies relevant to DUNE, however, $\mathcal{O}(1 \mathrm{GeV})$, the shower may not be developed, as we have seen here.

Our findings have two major applications. First, they can be directly applied to the analysis of the test-beam ProtoDUNE data. The comparison should make it possible to validate the parameters of the simulation framework, as well as help guide the analysis of the experimental data.

Second, they have implications for how the physics reach of liquid argon experiments is assessed. In situations where one is interested in general estimates of sensitivity to beyond the Standard Model scenarios, it may be acceptable 
to approximate the detector response with simple Gaussian errors. However, when accurate modeling is required-for example, in studying sensitivity to specific oscillation parameters-detailed, realistic models of the near and far detector are required for the results to be credible.

We hope that the present study will help with constructing such detailed models.

\section{ACKNOWLEDGMENTS}

We are grateful to Flavio Cavanna and Yun-Tse Tsai for useful discussions. We owe special thanks to the FLUKA development team for their support with the package. We are both supported by the U.S. Department of Energy under Contract No. DE-AC02-76SF00515.
[1] B. Abi et al. (DUNE Collaboration), arXiv:2002.03005.

[2] A. Friedland and S. W. Li, Phys. Rev. D 99, 036009 (2019).

[3] B. Abi et al. (DUNE Collaboration), arXiv:2007.06722.

[4] R. Acciarri et al. (DUNE Collaboration), arXiv:1512.06148.

[5] R. Acciarri et al. (DUNE Collaboration), arXiv:1601.02984.

[6] T. T. Böhlen, F. Cerutti, M. P. W. Chin, A. Fassò, A. Ferrari, P. G. Ortega, A. Mairani, P. R. Sala, G. Smirnov, and V. Vlachoudis, Nucl. Data Sheets 120, 211 (2014).

[7] A. Ferrari, P. R. Sala, A. Fassò, and J. Ranft, FLUKA: A multi-particle transport code; CERN-2005-010; SLAC-R773; INFN-TC-05-11.

[8] R. Acciarri et al. (ArgoNeuT Collaboration), Phys. Rev. D 99, 012002 (2019).

[9] M. Sorel, J. Instrum. 9, P10002 (2014).

[10] V. De Romeri, E. Fernandez-Martinez, and M. Sorel, J. High Energy Phys. 09 (2016) 030.
[11] N. Grant and T. Yang, in DPF meeting (2017), https://indico .fnal.gov/event/11999/contribution/275.

[12] W. Castiglioni, W. Foreman, I. Lepetic, B. Littlejohn, M. Malaker, and A. Mastbaum, arXiv:2006.14675.

[13] MicroBooNE Collaboration, Study of reconstructed 39Ar beta decays at the MicroBooNE detector, MICROBOONENOTE-1050-PUB, 2018, https://doi.org/10.2172/1573057.

[14] B. Bhandari et al. (CAPTAIN Collaboration), Phys. Rev. Lett. 123, 042502 (2019).

[15] We thank Flavio Cavanna for bringing this possibility to our attention.

[16] A. M. Ankowski and A. Friedland, Phys. Rev. D 102, 053001 (2020).

[17] A. M. Ankowski, A. Friedland, S. W. Li, O. Moreno, P. Schuster, N. Toro, and N. Tran, Phys. Rev. D 101, 053004 (2020). 